\title{
New Clustering Algorithm for Vector Quantization using Walsh Sequence
}

\author{
H. B. Kekre \\ Professor \\ Computer Engineering \\ Mukesh Patel School of \\ Technology Management and \\ Engineering, \\ NMIMS University, Vileparle(w) \\ Mumbai 400-056, India
}

\author{
Tanuja K. Sarode \\ Associate Professor \\ Computer Engineering, \\ Thadomal Shahani Engineering \\ College, \\ Bandra(W), Mumbai 400-050, \\ India
}

\author{
Jagruti K. Save \\ Ph.D. Scholar \\ Computer Engineering \\ Mukesh Patel School of \\ Technology Management and \\ Engineering, \\ NMIMS University, Vileparle(w) \\ Mumbai 400-056, India
}

\begin{abstract}
In this paper we present an effective clustering algorithm to generate codebook for vector quantization (VQ). Constant error is added every time to split the clusters in LBG, resulting in formation of cluster in one direction which is $135^{\circ}$ in $2-$ dimensional case. Because of this reason clustering is inefficient resulting in high MSE in LBG. To overcome this drawback of LBG proportionate error is added to change the cluster orientation in KPE. Though the cluster orientation in KPE is changed, its variation is limited to $\pm 45^{\circ}$ over $135^{\circ}$. KEVR introduces new orientation every time to split the clusters. But in KEVR the error vector sequence is the binary representation of numbers, so the cluster orientation change slowly in every iteration. To overcome this drawback we propose the technique which uses Walsh sequence to rotate the error vector. The proposed technique (Kekre's error vector rotation using Walsh - KEVRW) is based on KEVR algorithm. The proposed methodology is tested on different training images for code books of sizes 128, 256, 512, 1024. Our result shows that KEVRW gives less MSE and high PSNR compared to LBG, KPE and KEVR.
\end{abstract}

\section{General Terms}

Image Processing, Vector Quantization, Data Compression.

\section{Keywords}

Codebook, Code vector, Encoding, Walsh Function, Codebook Generation Algorithm, Image Compression.

\section{INTRODUCTION}

The fundamental goal of image compression is to reduce the number of bits required to store and transmit the image as well as to maintain the quality of the image. Several compression techniques have been developed such as vector quantization (VQ) [1][2], block truncation coding[3], transform coding, hybrid coding, and various adaptive versions of these methods. Among these techniques, vector quantization is widely used in image compression owing to its simple structure and low bit rate. Many types of VQ, such as classified VQ [4], address VQ [5], side match VQ [6], and predictive classified VQ [7] have been used for various purpose. VQ is widely used in many applications such as pattern recognition [8], face detection [9], image segmentation [10] [11], speech data compression [12], Content Based Image Retrieval (CBIR) [13], tumor detection in mammography images [14] etc. Vector quantization is a lossy image compression technique. VQ can be defined as a mapping function that maps $\mathrm{k}$ dimensional vector space to a finite set $\mathrm{CB}=\left\{\mathrm{C}_{1}, \mathrm{C}_{2}, \mathrm{C}_{3}, \ldots, \mathrm{C}_{\mathrm{N}}\right\}$. The set $\mathrm{CB}$ is called codebook consisting of $\mathrm{N}$ number of code vectors and each code vector $\mathrm{Ci}=\left\{\mathrm{c}_{\mathrm{i} 1}, \mathrm{c}_{\mathrm{i} 2}, \mathrm{c}_{\mathrm{i} 3}, \ldots, \mathrm{c}_{\mathrm{ik}}\right\}$ is of dimension $\mathrm{k}$. There are three main steps in vector quantization process: 1. Code book generation. 2. Encoding procedure 3. Decoding procedure. In Encoding phase image is divided into non overlapping blocks and each block then converted to the training vector $X_{i}=\left\{x_{i 1}\right.$, $\left.\mathrm{x}_{\mathrm{i} 2}, \ldots \ldots, \mathrm{x}_{\mathrm{ik}}\right\}$. The codebook is then searched for the nearest code vector $\mathrm{C}_{\min }$ by computing squared Euclidean distance as presented in equation (1) with vector $X_{i}$ with all the code vectors of the codebook $\mathrm{CB}$. Then for each input vector the index of its nearest code vector transmitted to the receiver. In the decoding procedure, an index is replaced by the corresponding code vector to reconstruct the encoded image at the receiver.

$$
\begin{aligned}
& d\left(X_{i}, C_{\min }\right)=\min _{1<=j<=N}\left\{d\left(X_{i}, C_{j}\right)\right\} \\
& \text { where } \\
& d\left(X_{i}, C_{j}\right)=\sum_{p=1}^{k}\left(X_{i p}-C_{j p}\right)^{2}
\end{aligned}
$$

A variety of encoding methods are available in literature like Partial Distortion search (PDS)[15], Mean-distance-ordered Partial Codebook Search Algorithm (MPS) [16], nearest neighbor search algorithm based on orthonormal transform (OTNNS) [17], Kekre's fast search algorithm [18] etc. The most important task for the vector quantization techniques is to design a good codebook [19]. To generate codebook there are various algorithms. The generalized Lloyd clustering algorithm (referred to as the LBG algorithm) [20] proposed by Linde, Buzo, and Gray is the most popular method for codebook generation. In this paper we present the codebook generation algorithm which is a modification to Kekre's error vector rotation algorithm (KEVR). We have used walsh sequence to generate the error matrix. The paper also compares the proposed algorithm with LBG, Kekre's proportionate error algorithm (KPE) [21], Kekre's error vector rotation algorithm (KEVR) [22], with respect to mean squared error (MSE) and peak signal to noise ratio (PSNR). In the next section we discuss LBG, KPE, KEVR algorithms. Section 3 gives some information on Walsh sequence. Proposed methodology is explained in Section 4, followed by results and conclusion in sections 5 and 6 respectively. 


\section{CODEBOOK GENERATION ALGORITHM}

\subsection{Linde, Buzo and Gray (LBG) Algorithm [20]}

The LBG algorithm is an iterative procedure. Starting with an initial codebook, the LBG algorithm performs the clustering of the training set, where an exhaustive search procedure is conducted to classify each vector in the training set according to its corresponding closest codeword in current codebook. The criteria to decide the closeness is squared Euclidean distance between the code vector and the training vector. Then each training vector records the index of its closest codeword in the codebook. After completing the clustering process for the whole training set, each codeword in the current codebook is updated with the centroid of those training vectors so that the current codeword is the closest codeword of them. The newly generated codebook is then used in the next iteration to minimize the overall averaged distortion in the codebook design procedure. Both the clustering and the updating procedures are executed repeatedly until the desired size of code book formed.

\subsection{Kekre's Proportionate Error Algorithm (KPE) [21]}

Here to generate two code vectors $\mathrm{C}_{1} \& \mathrm{C}_{2}$, proportionate error is added to the code vector. Magnitude of elements of the code vector decides the error ratio. Hereafter the procedure is same as that of LBG. While adding proportionate error a safe guard is also introduced so that neither v1 nor v2 go beyond the training vector space.

\subsection{Kekre's Error Vector Rotation Algorithm (KEVR) [22]}

In this algorithm two code vectors $\mathrm{C}_{1} \& \mathrm{C}_{2}$ are generated by adding and subtracting error vector rotated in $\mathrm{k}$-dimensional space at different angle. Error vector $e_{i}$ is the $i^{\text {th }}$ row of the error matrix of dimension $\mathrm{k}$. The error vectors matrix $\mathrm{E}$ is given in Equation (2).

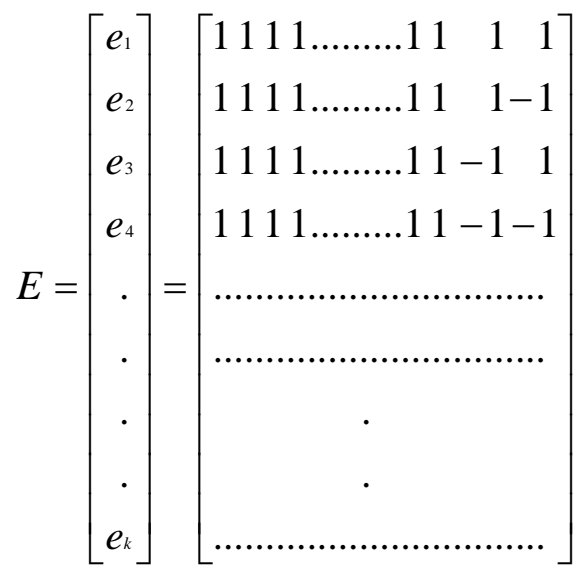

The error vector sequence have been obtained by taking binary representation of numbers starting from 0 to k-1 and replacing 0 's by 1 's and 1's by -1 's.

\section{WALSH SEQUENCE}

Walsh functions have become quite useful in the applications of image processing [23]. Walsh functions were established as a set of normalized orthogonal functions, analogous to sine and cosine functions, but having uniform values \pm 1 throughout their segments [24]. The Walsh functions are closely related to binary sequences, and they are, by definition, of length $2^{\mathrm{n}}$. These Walsh sequences are generated by sampling Walsh functions at the center of the interval. Walsh matrix is symmetrical. The first $8 \mathrm{Walsh}$ sequences are given in Figure 1.

\begin{tabular}{|l|l|l|l|l|l|l|l|}
\hline 1 & 1 & 1 & 1 & 1 & 1 & 1 & 1 \\
\hline 1 & 1 & 1 & 1 & -1 & -1 & -1 & -1 \\
\hline 1 & 1 & -1 & -1 & -1 & -1 & 1 & 1 \\
\hline 1 & 1 & -1 & -1 & 1 & 1 & -1 & -1 \\
\hline 1 & -1 & -1 & 1 & 1 & -1 & -1 & 1 \\
\hline 1 & -1 & -1 & 1 & -1 & 1 & 1 & -1 \\
\hline 1 & -1 & 1 & -1 & -1 & 1 & -1 & 1 \\
\hline 1 & -1 & 1 & -1 & 1 & -1 & 1 & -1 \\
\hline
\end{tabular}

Figure 1: First 8 Walsh sequences

\section{PROPOSED ALGORITHM (Kekre's Error Vector Rotation using Walsh Sequence - KEVRW)}

In this algorithm image is divided into non overlapping blocks. Each block is converted into training vector of dimension $\mathrm{k}$. Initially all vectors are considered to be in one cluster. Its centroid represents first code vector. Generate ' $n$ ' Walsh sequences where code book size of $2^{\mathrm{n}}$ is required by sampling Walsh functions at the center of interval. Since each sequence is symmetric from the center, crop the sequence from both sides equally such that the length of the sequence is $\mathrm{k}$. Then add and subtract first Walsh sequence to the first code vector to generate two code vectors. Calculate Euclidean distance between the training vectors in the cluster and the code vectors to split the cluster into two. Update the cluster centroids. In the next iteration, add and subtract next Walsh sequence to the cluster centroids. The procedure is repeated for each cluster till the code book of desired size is obtained.

\section{RESULTS}

The algorithms discussed above are implemented using MATLAB 7.0 on Pentium IV, $1.66 \mathrm{GHz}, 1 \mathrm{~GB}$ RAM. To test the performance of these algorithms ten color images as shown in Figure 2 belonging to different classes are used. The images used belong to class Animal, Bird, Monument, Vehicle, Fruits, and Scenery etc. Image is divided into 2 x 2 blocks, so each training vector is of dimension 12. To implement KEVRW (proposed algorithm), Walsh sequences of length 16 is generated and cropped by two columns from both sides to generate the Walsh sequence of dimension 12 . Figure 3 shows the results of LBG, KPE, KEVR and Proposed algorithm (KEVRW) from codebook size 1024 on Baboon image. It can be seen that the LBG method loses color and sharpness in the image. KPE and KEVR gives better results compare to LBG. The proposed technique-KEVRW retains color and the sharpness as compared to KPE and KEVR. Table 1 shows the comparison of LBG, KPE, KEVR and KEVRW for codebook sizes 128 and 256 with respect to MSE, PSNR for the training images. Table 2 shows the comparison of LBG, KPE, KEVR and KEVRW for codebook sizes 512 and 1024 with respect to MSE, PSNR for the training images. Figure 4 shows the average MSE performance for LBG, KPE, KEVR and KEVRW for different code book $(\mathrm{CB})$ sizes. 

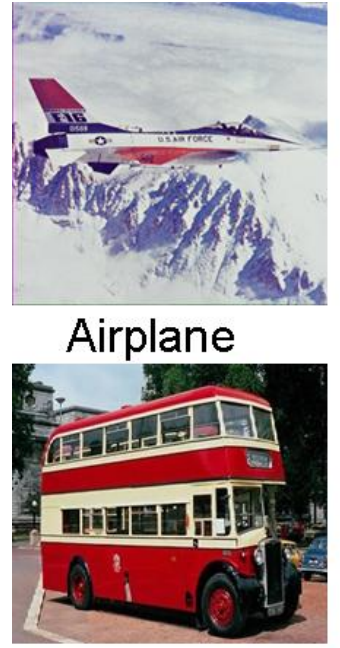

Bus

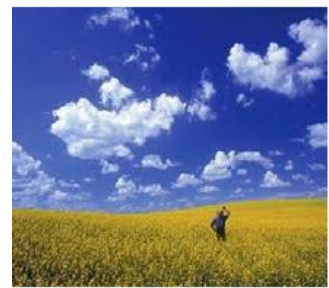

Scene

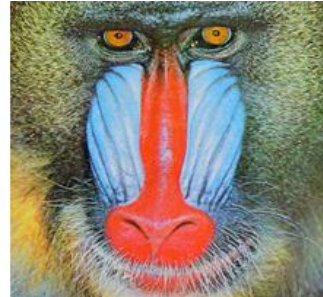

Baboon

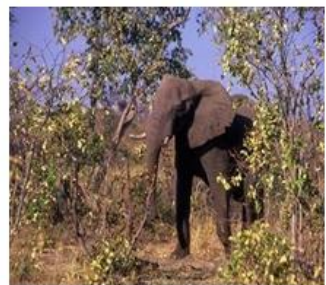

Elephant

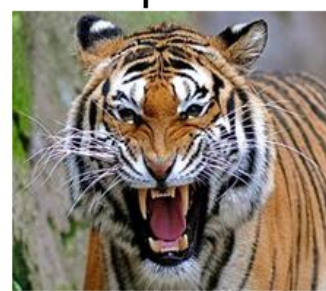

Tiger

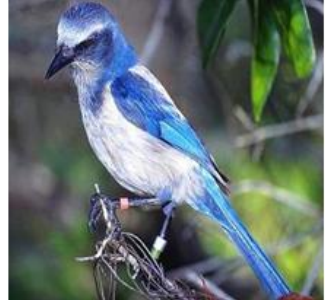

Bird

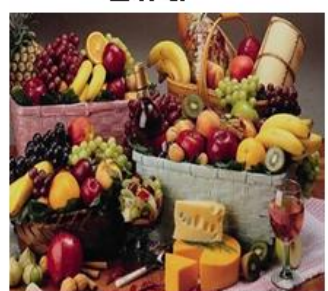

Fruits

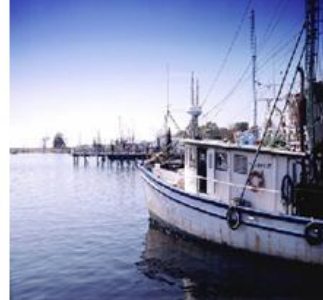

Boat

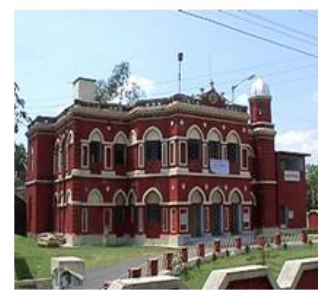

Mahal

Figure 2: Training Images

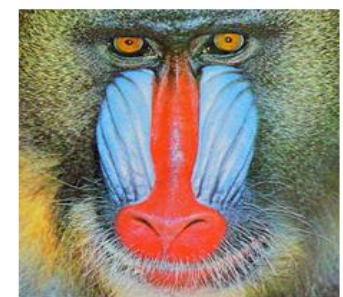

Baboon

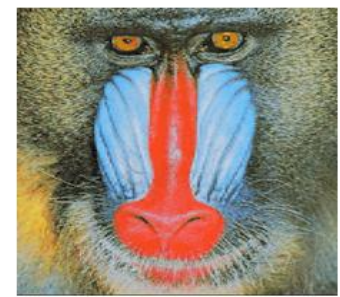

KEVR (Mse=205.60)

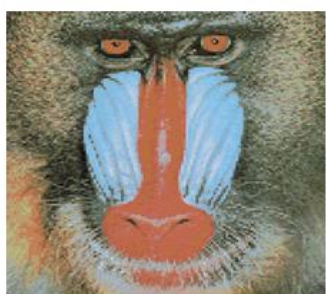

LBG (Mse=581.85)

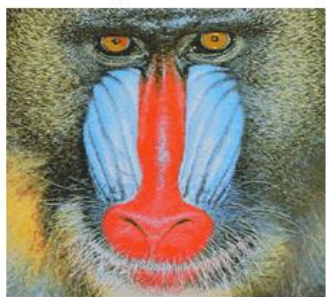

KEVRW (Mse=166.12)

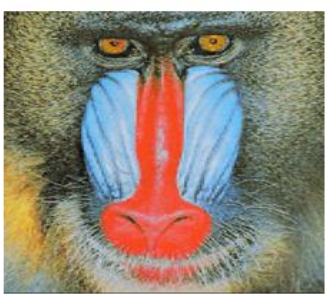

KPE (Mse=225.08)

Figure 3: Results of LBG, KPE, KEVR and KEVRW from codebook size 1024 on Baboon image 
Table 1. Comparison of LBG, KPE, KEVR AND KEVRW for codebook size 128 and 256 with respect to MSE, PSNR for the training images.

\begin{tabular}{|c|c|c|c|c|c|c|c|c|c|}
\hline \multirow[t]{2}{*}{ Images } & \multirow{2}{*}{$\begin{array}{c}\text { Param- } \\
\text { eters }\end{array}$} & LBG & KPE & KEVR & KEVRW & LBG & KPE & KEVR & KEVRW \\
\hline & & \multicolumn{4}{|c|}{128} & \multicolumn{4}{|c|}{256} \\
\hline \multirow[t]{2}{*}{ Airplane } & MSE & 221.63 & 189.22 & 127.96 & 99.44 & 201.96 & 139.96 & 100.81 & 71.68 \\
\hline & PSNR & 24.67 & 25.36 & 27.06 & 28.16 & 25.08 & 26.67 & 28.10 & 29.58 \\
\hline \multirow[t]{2}{*}{ Baboon } & MSE & 956.30 & 527.00 & 400.06 & 359.91 & 875.22 & 399.62 & 352.45 & 299.66 \\
\hline & $\overline{\text { PSNR }}$ & 18.32 & 20.91 & 22.11 & 22.57 & 18.71 & 22.11 & 22.66 & 23.36 \\
\hline \multirow[t]{2}{*}{ Bird } & MSE & 497.08 & 338.80 & 231.07 & 212.59 & 444.97 & 258.67 & 190.18 & 161.26 \\
\hline & PSNR & 21.17 & 22.83 & 24.49 & 24.86 & 21.65 & 24.00 & 25.34 & 26.06 \\
\hline \multirow[t]{2}{*}{ Boat } & MSE & 337.88 & 320.94 & 192.25 & 156.15 & 314.84 & 257.13 & 154.69 & 112.49 \\
\hline & PSNR & 22.84 & 23.07 & 25.29 & 26.20 & 23.15 & 24.03 & 26.24 & 27.62 \\
\hline \multirow[t]{2}{*}{ Bus } & MSE & 652.57 & 521.07 & 338.14 & 225.24 & 584.22 & 319.99 & 266.43 & 163.59 \\
\hline & PSNR & 19.98 & 20.96 & 22.84 & 24.60 & 20.47 & 23.08 & 23.87 & 25.99 \\
\hline \multirow[t]{2}{*}{ Elephant } & MSE & 726.53 & 537.02 & 387.68 & 316.49 & 659.86 & 370.48 & 339.08 & 267.21 \\
\hline & PSNR & 19.52 & 20.83 & 22.25 & 23.13 & 19.94 & 22.44 & 22.83 & 23.86 \\
\hline \multirow[t]{2}{*}{ Fruits } & MSE & 530.54 & 441.87 & 261.84 & 255.94 & 485.33 & 314.84 & 223.01 & 204.20 \\
\hline & PSNR & 20.88 & 21.68 & 23.95 & 24.05 & 21.27 & 23.15 & 24.65 & 25.03 \\
\hline \multirow[t]{2}{*}{ Mahal } & MSE & 468.22 & 425.20 & 283.35 & 216.31 & 427.48 & 298.82 & 230.56 & 166.30 \\
\hline & PSNR & 21.43 & 21.84 & 23.61 & 24.78 & 21.82 & 23.38 & 24.50 & 25.92 \\
\hline \multirow[t]{2}{*}{ Scene } & MSE & 704.74 & 199.80 & 161.14 & 131.17 & 592.29 & 170.38 & 135.67 & 103.02 \\
\hline & PSNR & 19.65 & 25.12 & 26.06 & 26.95 & 20.41 & 25.82 & 26.81 & 28.00 \\
\hline \multirow[t]{2}{*}{ Tiger } & MSE & 658.10 & 579.90 & 383.37 & 344.06 & 605.89 & 425.52 & 325.49 & 256.51 \\
\hline & PSNR & 19.95 & 20.50 & 22.29 & 22.76 & 20.31 & 21.84 & 23.01 & 24.04 \\
\hline \multirow[t]{2}{*}{ Average } & MSE & 575.36 & 408.08 & 276.69 & 231.73 & 519.21 & 295.54 & 231.84 & 180.59 \\
\hline & PSNR & 20.84 & 22.31 & 23.99 & 24.80 & 21.28 & 23.65 & 24.80 & 25.95 \\
\hline
\end{tabular}

\section{Mean Squared Error}

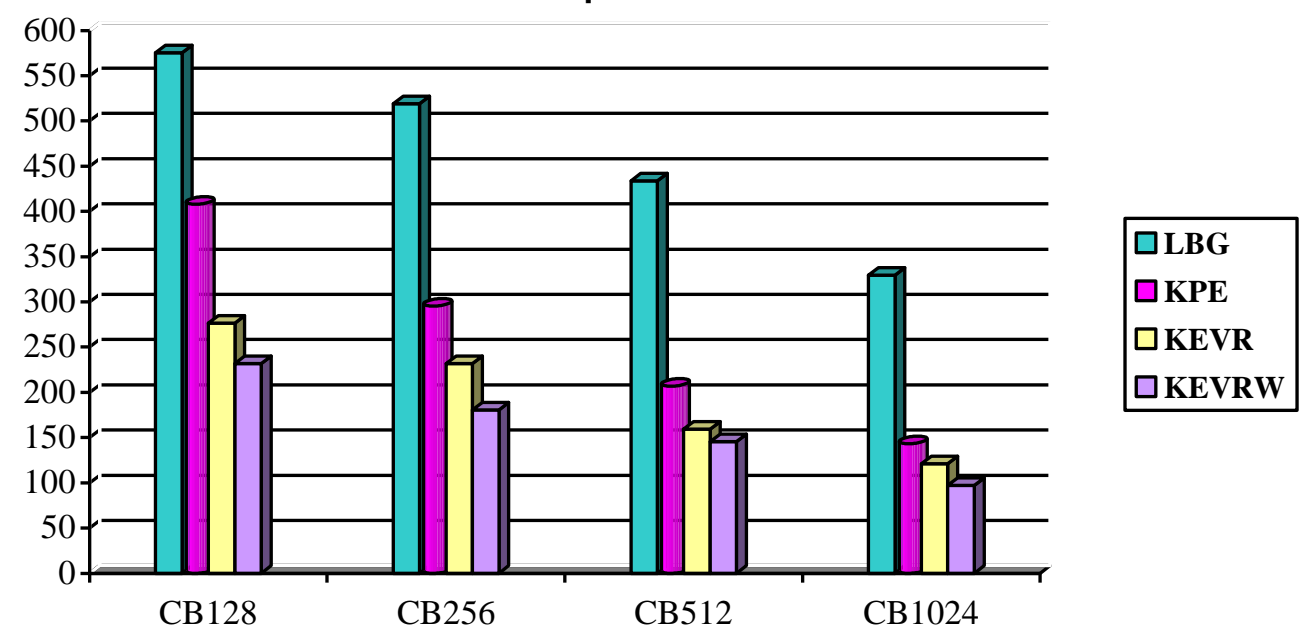

Figure 4 Average MSE performances for LBG, KPE, KEVR and KEVRW for different code book (CB) sizes 
Table 2. Comparison of LBG, KPE, KEVR AND KEVRW for codebook size 512 and 1024 with respect to MSE, PSNR for the training images.

\begin{tabular}{|c|c|c|c|c|c|c|c|c|c|}
\hline \multirow[t]{2}{*}{ Images } & \multirow[t]{2}{*}{$\begin{array}{l}\text { Paramet } \\
\text { ers }\end{array}$} & LBG & KPE & KEVR & $\begin{array}{l}\text { KEVR } \\
\text { W }\end{array}$ & LBG & KPE & KEVR & KEVRW \\
\hline & & \multicolumn{4}{|c|}{512} & \multicolumn{4}{|c|}{1024} \\
\hline \multirow[t]{2}{*}{ Airplane } & MSE & 171.74 & 94.29 & 68.34 & 56.06 & 131.90 & 58.68 & 48.82 & 38.26 \\
\hline & PSNR & 25.78 & 28.39 & 29.78 & 30.64 & 26.93 & 30.45 & 31.24 & 32.30 \\
\hline \multirow[t]{2}{*}{ Baboon } & MSE & 743.28 & 303.05 & 261.26 & 246.23 & 581.85 & 225.08 & 205.60 & 166.12 \\
\hline & PSNR & 19.42 & 23.32 & 23.96 & 24.22 & 20.48 & 24.61 & 25.00 & 25.93 \\
\hline \multirow[t]{2}{*}{ Bird } & MSE & 373.50 & 199.64 & 139.27 & 127.90 & 297.20 & 137.63 & 104.60 & 84.64 \\
\hline & PSNR & 22.41 & 25.13 & 26.69 & 27.06 & 23.40 & 26.74 & 27.94 & 28.85 \\
\hline \multirow[t]{2}{*}{ Boat } & MSE & 272.06 & 164.43 & 93.09 & 90.16 & 220.35 & 97.20 & 68.63 & 58.34 \\
\hline & PSNR & 23.78 & 25.97 & 28.44 & 28.58 & 24.70 & 28.25 & 29.77 & 30.47 \\
\hline \multirow[t]{2}{*}{ Bus } & MSE & 496.00 & 191.49 & 141.91 & 130.39 & 380.52 & 122.59 & 105.92 & 86.13 \\
\hline & PSNR & 21.18 & 25.31 & 26.61 & 26.98 & 22.33 & 27.25 & 27.88 & 28.78 \\
\hline \multirow[t]{2}{*}{ Elephant } & MSE & 572.23 & 285.72 & 254.71 & 217.51 & 434.49 & 214.08 & 200.29 & 142.27 \\
\hline & PSNR & 20.56 & 23.57 & 24.07 & 24.76 & 21.75 & 24.83 & 25.11 & 26.60 \\
\hline \multirow[t]{2}{*}{ Fruits } & MSE & 417.74 & 222.99 & 168.64 & 166.88 & 331.92 & 167.24 & 134.54 & 117.82 \\
\hline & PSNR & 21.92 & 24.65 & 25.86 & 25.91 & 22.92 & 25.90 & 26.84 & 27.42 \\
\hline \multirow[t]{2}{*}{ Mahal } & MSE & 359.58 & 185.20 & 136.05 & 134.45 & 269.75 & 123.21 & 98.63 & 86.91 \\
\hline & PSNR & 22.57 & 25.45 & 26.79 & 26.85 & 23.82 & 27.22 & 28.19 & 28.74 \\
\hline \multirow[t]{2}{*}{ Scene } & MSE & 396.12 & 132.97 & 102.37 & 82.62 & 220.45 & 91.69 & 75.72 & 55.17 \\
\hline & PSNR & 22.15 & 26.89 & 28.03 & 28.96 & 24.70 & 28.51 & 29.34 & 30.71 \\
\hline \multirow[t]{2}{*}{ Tiger } & MSE & 535.70 & 288.07 & 228.15 & 204.68 & 424.77 & 194.96 & 166.40 & 133.80 \\
\hline & PSNR & 20.84 & 23.54 & 24.55 & 25.02 & 21.85 & 25.23 & 25.92 & 26.87 \\
\hline \multirow[t]{2}{*}{ Average } & MSE & 433.80 & 206.79 & 159.38 & 145.69 & 329.32 & 143.24 & 120.91 & 96.95 \\
\hline & PSNR & 22.06 & 25.22 & 26.48 & 26.90 & 23.29 & 26.90 & 27.72 & 28.67 \\
\hline
\end{tabular}

\section{CONCLUSIONS}

In this paper a codebook generation algorithm using Walsh sequence is proposed and compared with traditional LBG, KPE and KEVR. This paper aims to present an improvement to KEVR algorithm. In KEVR, only one digit of error vector changes in each iteration, which results in slowly changing cluster orientation. This drawback is removed in proposed technique-KEVRW. In KEVRW, the Walsh sequence is used to generate error vector. The Walsh sequences are symmetric in nature and half the number of digits change in successive Walsh sequences. So there is a fast change in cluster orientation. This gives effective clustering. It is observed that KPE and KEVR give far better performance as compared to LBG algorithm. However the proposed new algorithm KEVRW improves this performance. The proposed method reduces MSE by $59 \%$ to $70 \%$ for codebook size 128 to 1024 with respect to LBG, by $43 \%$ to $32 \%$ with respect to KPE and by $16 \%$ to $19 \%$ with respect to KEVR. It is observed that more error reduction is obtained for images like Baboon and Bus. It can be concluded that the images having more edges will give more reduction of error.

\section{REFERENCES}

[1] A. Gersho, R. M. Gray, "Vector Quantization and Signal Compression", Kluwer Academic Publishers, Boston, MA, 1991

[2] R. M. Gray, "Vector quantization", IEEE ASSP Mag, Apr.1984

[3] H. B. Kekre and S. D. Thepade, "Image Retrieval using Augmented Block Truncation Coding Techniques", ACM International Conference on Advances in Computing, Communication and Control (ICAC3-2009), pp. 384-390, Jan 2009, Fr. Conceicao Rodrigous College of Engg., Mumbai.

[4] Y. C. Liaw, J. Z. C. Lai, and W. Lo, "Image Restoration of Compressed Image using Classified Vector Quantization",Pattern Recogn. vol. 35, No.2, pp. 181192, 2002.

[5] N.M. Nasrabadi and Y. Feng, "Image Compression using Address Vector Quantization", IEEE Trans. Commun., vol. 38, No. 12, pp. 2166-2173, 1990.

[6] T. Kim, "Side Match and Overlap Match Vector Quantizers for Images", IEEE Trans. Image Process., vol. 1, No. 2, pp.170-185, 1992. 
[7] K.N. Ngan and H.C. Koh, "Predictive Classified Vector Quantization", IEEE Trans. Image Process. vol. 1, No. 3, pp. 269-280, 1992.

[8] A. A. Abdelwahab and N. S. Muharram, "A Fast Codebook Design Algorithm Based on a Fuzzy Clustering Methodology", International Journal of Image and Graphics, vol. 7, No. 2, pp. 291-302, 2007.

[9] C. Garcia and G. Tziritas, "Face Detection using Quantized Skin Color Regions Merging and Wavelet Packet Analysis", IEEE Trans. Multimedia, vol. 1, No. 3, pp. 264-277, Sep. 1999

[10] H. B. Kekre, T. K. Sarode and B. Raul, "Color Image Segmentation using Kekre's Algorithm for Vector Quantization", International Journal of Computer Science (IJCS), vol. 3,No. 4, pp. 287-292, Fall 2008.

[11] H. B. Kekre, T. K. Sarode and B. Raul, "Color Image Segmentation using Vector Quantization Techniques Based on Energy Ordering Concept", International Journal of Computing Science and Communication Technologies (IJCSCT), vol. 1, Issue 2, January 2009.

[12] H. B. Kekre and T. K. Sarode, "Speech Data Compression using Vector Quantization", WASET, International Journal of Computer and Information Science and Engineering, (IJECSE), vol. 2, Number 4, pp. 251-254, 2008.

[13] H. B. Kekre, T. K. Sarode and S. D. Thepade, "Image Retrieval using Color-Texture Features from DCT on VQ Codevectors obtained by Kekre's Fast Codebook Generation", ICGST-International Journal on Graphics, Vision and Image Processing (GVIP), vol. 9, Issue 5, pp. 1-8, September 2009.

[14] H. B. Kekre, T. K. Sarode and S. Gharge, "Detection and Demarcation of Tumor using Vector Quantization in MRI images," International Journal of Engineering Science and Technology, vol.1, Number 2, pp. 59-66, 2009.

[15] C. D. Bei and R. M. Gray, "An Improvement of the Minimum Distortion Encoding Algorithm for Vector Quantization", IEEE Trans. Commun., vol. 33, No. 10, pp. 1132-1133, Oct. 1985.

[16] S. W. Ra, and J. K. Kim, "A Fast Mean-DistanceOrdered Partial Codebook Search Algorithm for Image Vector Quantization", IEEE Trans. on Circuits and Systems-11,Analog and Digital Signal Processing, vol. 40, No. 9, pp. 576-579, 1993.

[17] Z. Li, and Z.- M. Lu, "Fast Codevector Search Scheme for 3D Mesh Model Vector Quantization", Electron. Lett., vol. 44, No. 2, pp. 104-105, Jan 2008.

[18] H. B. Kekre and T. K. Sarode, "Centroid Based Fast Search Algorithm for Vector Quantization", International Journal of Imaging (IJI), vol. 1, No. 08, pp. 73-83, Autumn 2008

[19] C. M. Huang and R. W. Harris, "A Comparison of Several Vector Quantization Codebook Generation Approaches", IEEE Trans. On Image Processing, vol. 2, No. 1, pp. 108-112, 1993

[20] Y. Linde, A. Buzo, and R. M. Gray, "An Algorithm for Vector Quantizer Design", IEEE Trans. Commun., vol. COM-28, No. 1, pp. 84-95, 1980

[21] H. B. Kekre and T. K. Sarode, "New Clustering Algorithm for Vector Quantization using Rotation of Error Vector", International Journal of Computer Science and Information Security,(IJCSIS), vol. 7, No. 3, pp. 159-165, 2010.
[22] H. B. Kekre and T. K. Sarode, "Clustering Algorithm for codebook Generation using Vector Quantization", National Conference on Image Processing, TSEC, India, Feb 2005 .

[23] H.B.Kekre and D. Mishra, "Density Distribution and Sector Mean with Zero-Sal and Highest-Cal Components in Walsh transform Sectors as Feature Vectors for Image Retrieval", International Journal of Computer Scienece and Information Security (IJCSIS), vol.8, No. 4, 2010, ISSN 1947-5500

[24] J.L.Walsh, "A Closed Set of Orthogonal Functions", American Journal of Mathematics, vol. 45, pp. 5-24, 1923.

\section{AUTHORS PROFILE}

Dr. H. B. Kekre has received B.E. (Hons.) in Telecomm. Engineering. from Jabalpur University in 1958, M.Tech (Industrial Electronics) from IIT Bombay in 1960, M.S.Engg. (Electrical Engg.) from University of Ottawa in 1965 and $\mathrm{Ph} . D$. (System Identification) from IIT Bombay in $1970 \mathrm{He}$ has worked as Faculty of Electrical Engineering and then HOD Computer Science and Engg. at IIT Bombay. For 13 years he was working as a professor and head in the Department of Computer Engg. at Thadomal Shahani Engineering. College, Mumbai. Now he is Senior Professor at MPSTME, SVKM's NMIMS University. He has guided 17 Ph.Ds, more than 100 M.E./M.Tech and several B.E./ B.Tech projects. His areas of interest are Digital Signal processing, Image Processing and Computer Networking. He has more than 450 papers in National /International Conferences and Journals to his credit. He was Senior Member of IEEE. Presently He is Fellow of IETE and Life Member of ISTE Recently twelve students working under his guidance have received best paper awards. Currently 7 research scholars are pursuing $\mathrm{Ph} . \mathrm{D}$. program under his guidance.

Tanuja K. Sarode has Received Bsc. (Mathematics) from Mumbai University in 1996, Bsc.Tech. ( Computer Technology) from Mumbai University in 1999, M.E. (Computer Engineering) from Mumbai University in 2004, currently Pursuing Ph.D. from Mukesh Patel School of Technology, Management and Engineering, SVKM's NMIMS University, Vile-Parle (W), Mumbai, INDIA. She has more than 10 years of experience in teaching. She is currently working as Associate Professor in Dept. of Computer Engineering at Thadomal Shahani Engineering College, Mumbai. She is life member of IETE, member of International Association of Engineers (IAENG) and International Association of Computer Science and Information Technology (IACSIT), Singapore. Her areas of interest are Image Processing, Signal Processing and Computer Graphics. She has 100 papers in National /International Conferences/journal to her credit.

Jagruti K. Save has Received B.E. (Computer Engg.) from Mumbai University in 1996, M.E. (Computer Engineering) from Mumbai University in 2004, currently Pursuing Ph.D. from Mukesh Patel School of Technology, Management and Engineering, SVKM's NMIMS University, Vile-Parle (W), Mumbai, INDIA. She has more than 10 years of experience in teaching. Currently working as Associate Professor in Dept. of Computer Engineering at Fr. Conceicao Rodrigues College of Engg., Bandra, Mumbai. Her areas of interest are Image Processing, Neural Networks, Fuzzy systems, Data base management and Computer Vision. She has 5 papers in National/International Conferences to her credit. 